\title{
¿EXISTEN MOVIMIENTOS SOCIALES?
}

ANGELES DIEZ RODRIGUEZ

Esta reflexión es una propuesta analítica acerca del problema de "Ios movimientos sociales". Trata de cambiar, en parte, el ángulo de lectura de la realidad social que ha sido incluida en dicho concepto a la vez que ubicar la discusión teórica.

En los últimos años el desarrollo teórico en torno a los "movimientos sociales" parece haberse estancado, bien en la pura descripción de casos concretos, bien en la mera especulación sin apoyaturas reales. Es un fenómeno que está relacionado, desde mi punto de vista, con la brecha abierta entre conocimiento y práctica social.

Dicha desvinculación o desconexión tiene que ver con la inserción de los intelectuales en el aparato del poder (que dificulta, entre otras cosas, la capacidad de apreciar la realidad no integrada), y con la creciente complejización del sistema social que hace a muchos optar por el conocimiento ya consolidado o por la comodidad de los conceptos bien delimitados.

Este proceso ha dado lugar a dos tipos de pensamiento, uno desde posiciones aparentemente progresistas que ha quedado atrapado en la circularidad de cuestiones como: si son o no nuevos los movimientos sociales, si son o no transformadores, si son la alternativa a las clases sociales y a los partidos revolucionarios, cuáles son sus revindicaciones $y$ de qué forma podemos hacer una tipología y saberlo todo acerca de ellos; otro, que ni siquiera toma nota de la "novedad social" y declara "el fin de la historia".

Estamos ante una "crisis de paradigmas" que se expresa en las dificultades del conocimiento científico para orientar los procesos sociales, a la vez que, cada vez con mayor frecuencia, parece no ser capaz de nutrirse de la realidad en ebullición.

Esto ocurre porque lo real es siempre mucho más complejo que nuestro pensamiento, de ahí que los conceptos, lo mismo pueden ser nuestro pensamiento, de ahí que los conceptos, lo mismo pueden ser herramientas muy útiles que convertirse en obstáculos insalvables. Nos pueden ayudar a ver lo real o ser orejeras que impidan nuestra visión.

Como alguna vez apuntara Popper las grandes preguntas nacen fuera de lo que sabemos. De ahí que no sea vanal interrogarse sobre la realidad que queda incluida dentro del concepto de "movinientos sociales" y más aún cual es la cara oculta de la luna, es decir, que hay detrás de la pregunta: ¿qué son los movimientos sociales?.

Desde mi punto de vista, la discusión sobre los movimientos sociales es un debate sobre:

a) La integración social o consenso (en sus diferentes aspectos, cohesión, eficacia, legitimación, etc.),

b) Sobre la constitución de los sujetos de la acción social.

1. La integración y el disenso: Después de las "revoluciones socialistas" de los países del este y lo que a ellas siguió, la capacidad del capitalismo para sobrevivir a las crisis periódicas y la ausencia de lucha revolucioviolenta por parte de la clase obrera de los 
países del este y que lo que a ellas siguió, la capacidad del capitalismo para sobrevivir a las crisis periódicas y la ausencia de lucha revolucionaria violenta por parte de la clase obrera de los países de europa occidental, distintos autores marxistas se inclinaron hacia una nueva interpretación del capitalismo tardío que buscaba explicar la cohesión social en términos de integración ideológica de la fuerza de trabajo. El control se habría hecho tan sofisticado que las "clases subalternas" identificaban sus intereses con los del capitalismo y no con un movimiento revolucionario.

Los máximos representantes de esta interpretación fueron los componentes de la escuela de Francfort, aunque no fueron los primeros en valorar los factores superestructurales ya que marxistas como Gramsci reaccionaron contra el reduccionismo económico mucho antes. Para Marcuse, Adorno, Horheiner... la sociedad generada por el capitalismo tardío, la sociedad unidimensional en la que se había impuesto la racionalidad tecnológica, había hecho desaparecer al "Sujeto revolucionario" ya que no sólo la clase trabajadora estaba totaimente integrada sino que además la tendencia era a su desaparición dado el incremento de la automatización y el peso crecimiento de los "white collars". "La conciencia feliz", nos decía Marcuse," o sea, la creencia de que lo real es racional y el sistema social establecido produce los bienes, refleja un nuevo conformismo que se presenta como una faceta de la racionalidad tecnológica y se traduce en una forma de conducta social" (P.114).

De esta forma, para todos ellos que vivieron la caída de la efervescencia revolucionaria, el nazismo y la posterior sociedad consensualizada e integrada, el futuro se presentaba $\sin$ salida, $\sin$ alternativa al "bárbaro" capitalismo por ausencia de sujeto transformador- a pesar de que algunos intentaron buscarlo en la marginalidad, dado que la clase obrera no sería ya más nunca la vanguardia revolucionaria, o en las luchas del tercer mundo-. Como decía Jhon D. Galbraith, en American Capitalism: "iLa comunidad está demasiado satisfecha para preocurse! "refiriéndose a la sociedad norteamericana.

Fue en este contexto en el que estalló el Mayo del 68 refutando la tesis de la ideología dominante, de la cultura común y la integración social, que en parte seguían siendo deudoras de los "modelos objetivos" de clases sociales; matizándolos, desde luego, ya que rompían el hilo mecanicista que unia condiciones objetivas de existencia con unos intereses y una conciencia de clase.

Los sujetos transformadores nacían no sólo en la América Latina incapaz de resolver sus problemas económicos, sino en países de economía floreciente. Frente al cierre del universo en el occidente desarrollado que sostenían los francfortnianos, la apertura de una realidad en movimiento. También serían más tarde el movimiento pacifista, el ecologísta, la lucha barrial, etc. Su visión de la historia era, en el fondo, más weberiana que marxista al pensar en la capacidad del capitalismo para subsumir plenamente al individuo.

Con la aparición de esos "los movimientos sociales" de los 70 en los países desarrollados, se cuestionaba la integración social, pero además se ponían otras dos cuestiones sobre el tapete, la primera, acerca de las características de los sujetos que entraban en escena, la segunda, sobre las motivaciones.

Si el Mayo francés fue posible, a pesar de la supuesta adaptación e integración de la población en las sociedades desarrolladas, si los estudiantes se movilizaron bajo lemas marxistas que no acababan de comprender en profundidad al tiempo que revindicaban la fantasía, y si a ellos se sumaron las revindicaciones obreras, a pesar de que no existía una situación explosiva al interior del sistema capitalista sino que se trataba de una época de pleno empleo y de salarios al alza; decimos que, fue posible porque se 
demandaba mejoras de calidad de vida que el capitalismo negaba pero susceptibles de ser concedidas en esos momentos de relanzamiento del capital y una vez recuperados los desastres de la guerra (las posibilidades de asimilación eran grandes); por otro lado se vivían momentos de ascenso revolucionario en el "tercer mundo" a los que se sumaba el auge de paradigmas emancipatorios "humanistas" como los del Che o Ho Chi Minh.

No obstante, estas luchas anunciaban una dimensión más de la crisis del capitalismo tardío que no se había tomado en cuenta y que aparentemente no se relacionaba con el crecimiento y desarrollo económico sino con la enajenación y la insatisfacción de la cultura consumista ineludiblemente unida al capitalismo y con la falta de participación política. De qué forma estas movilizaciones sociales se relacionan o no con las del tercer mundo y cómo pueden servir para complejizar más el análisis de los movimientos latinoamericanos es algo que veremos más tarde. De hecho, fueron una llamada de atención respecto de la identidad de los sujetos de la acción social revolucionaria.

La influencia del Keynesianismo tras la Segunda Guerra Mundial inauguraba un período de redistribución de los excedentes a importantes sectores de la población. La conflictividad comenzaba a localizarse en espacios hasta entonces reservados a las clases altas (medio ambiente, calidad de vida, derechos de la mujer, etc), se daba una mayor participación económica de las clases medias y se demandaban espacios políticos de participación. Según el concepto de "legitimación" acuñado por Habermas, las clases dominadas aceptarían el sistema social por la acción combinada de la conciencia impuesta por las clases dominantes (ideología) y por la existencia de gratificaciones reales 0 recompensas materiales. El problema pues era otro, la fractura en el sistema de valores.

La última década, la de los ochenta, en las sociedades desarrolladas, no ha estado exenta de conflictividad social, y los cientistas sociales han seguido preocupados por "los nuevos movimientos sociales", según ellos cada vez más "nuevos". Pero, esta vez, se habla de la "Crisis del Estado de Bienestar" en cuanto a su capacidad Administrativa-Institucional, o lo que es lo mismo, los límites de la expansión "necesaria" del Estado en sus mecanismos de control social y de consenso que garanticen el funcionamiento eficaz del sistema.

Claus Offe representa una de las teorizaciones más consistentes al respecto. Cuando se preguntan por los "partidos políticos y los nuevos movimientos sociales" (op. cit. 198), su análisis discurre sobre dos coordenadas: los procesos de burocratización (racionalidad burocrático-formal al estilo weberiano), y la eficacia en el mantenimiento del orden socio-económico a partir de la legitimación y consenso. El problema de los movimientos sociales sigue siendo un problema institucional o de participación política. La búsqueda del consenso pasa por una mayor eficacia administrativa, por la articulación de canales de participación y por el reconocimiento de los actores revindicativos (cuya identidad no es tanto la clase social como el género, la raza, la edad, etc.). Sin duda es muy interesante la visión de la identidad colectiva en función de caracteres más permanentes, eso lo veremos más tarde, lo que aquí queremos resaltar es que la preocupación por los movimientos sociales está motivada por la no integración del individuo, es decir, por la percepción que tiene los científicos de los focos de resistencia. 
La competencia de partidos, símbolos de la democracia participativa habría ido - separando cada vez más la sociedad política de la sociedad civil, hasta tal punto que las identidades políticas de la sociedad civil no encuentran vías de participación por lo que recurren a la construcción de sus propios canales: nuevos movimientos sociales o asociaciones que utilizan la vía corporativista de negociación.

Parece claro que para los teóricos europeos, también para los políticos, la preocupación básica viene a ser recomposición de la racionalidad burguesa en el ámbito político institucional acorde a nuevos parámetros: con una mayor complejización que exigen los mecanismos del mercado cada vez menos sujetos a predictibilidad, cada vez más azarosos y con efectos perversos en sus regulaciones automáticas pues dejan fuera a sectores significativos como las clases medias.

Las movilizaciones en América Latina comienzan a ser interpretadas según estas claves (ver caso de Portantiero) como problemas de participación, implícitamente problemas de integración social.

Fuera ya de los intereses concretos de políticos y sociólogos, sale a la luz el hecho de que la base social de los movimientos europeos de la última década ha cambiado en parte. Sin embargo, la generalidad del término "movimiento social" impide discriminar la heterogeneidad de las realidades que incluye. Tampoco nos permite movernos con facilidad en el tiempo, al ser etiquetados de igual forma movimientos barriales, ecologístas, etc.

También son crecientes los esfuerzos por abandonar la interpretación en términos de clases sociales sustituyéndola por la idea de "movimientos sociales" cuyos contenidos se presentan ajenos a cualquier referencia a la estructura económico social (independientemente de si es o no determinante). Parece evidente que durante todos estos años la realidad se ha hecho más compleja y que es diferente, pero eso no es decir absolutamente nada acerca de la realidad. Por otro lado, si bien las extrapolaciones pueden resultar sugerentes y servirnos para añadir contenidos más ricos a los instrumentos de análisis no están ajenas a una intencionalidad política y a la posibilidad de un nuevo reducionismo esta vez en la esfera de lo superestructural.

2. La constitución de los sujetos de la acción soclal.- Este tema, que señalábamos como central en la discusión sobre movimientos sociales, es la otra cara de la moneda de la integración. Es el salto hacia la conciencia y práctica social en la elaboración de un proyecto de sociedad diferente (parcial o global).

La diversidad de manifestaciones y lugares en los que se expresan los movimientos sociales, así como su incapacidad para universalizar un proyecto impiden que podamos hablar de un "Sujeto histórico" por excelencia, aunque sí, podemos constatar que en determinados momentos de la historia (y dependiendo de las formas adoptadas por el capitalismo en los distintos espacios) ha habido un "centro estructurante" capaz de articular y ordenar la movilización social, nos referimos a las relaciones laborales.

Las condiciones de existencia unidas a la explotación de la mano de obra y los efectos directos de esa explotación fueron, en el occidente desarrollado, y son todavía, en los países latinoamericanos, el núcleo alrededor del cual las identidades colectivas elaboradas tienen mayores posibilidades de traducirse en transformaciones globales de la estructura social y económica (lo cual no significa que tenga que darse dicho salto 
necesariamente). Es por eso que movimiento obrero y movimientos urbanos populares forman una amalgama dificil de separar en el "tercer mundo".

En los países desarrollados, por el contrario, las transformaciones sufridas por el capitalismo y la materialización del Estado de Bienestar han debilitado la experiencia de la explotación directa. Es más, han hecho posible la distribución del excedente a una capa mayor de población. De ahí que, otros campos de la reproducción de la fuerza de trabajo (calidad de vida, tiempo libre etc...) sean ahora los referentes de la constitución de identidades colectivas, es decir, de "nuevos sujetos sociales". Las prácticas colectivas no tienen por escenario principal la fábrica y los lenguajes no son los de proletariado/capitalista. Eso no quiere decir que no se den movilizaciones obreras (en el espacio de la producción en sentido estricto), pero éstas, cada vez más, tienden a la corporativización y es fácil trazar una línea divisoria entre ellas y otro tipo de movilizaciones como las pacifistas o ecologístas.

Dejemos a un lado, por el momento, la diferenciación de los sujetos concretos y sus demandas, $y$ ascendamos un poco hacia lo que nos parece la esencia del conflicto social en las sociedades capitalistas. Nos referimos a la oposición que deviene de la incompatibilidad entre economía privada $y$ participación política de las masas. Es lo que hace que muchos investigadores unan el tema de los "movimientos sociales" al de la democracia.

La económia privada, regida por las leyes del mercado, se opone en esencia a los principios de igualdad social que presupone el sistema democrático participativo y eso es mucho más evidente en los países de América Latina por lo que la democracia encuentra dificultades para asentarse, tal vez por la tradición de tomarse al pie de la letra la concordancia entre democracia y bienestar social. El mercado representa la esfera de lo privado mientras que la política es la encarnación de lo público, el primero se guía por principios de competencia e individualidad, en el segundo ha de primar el interés colectivo y la igualdad.

Desde la segunda posguerra, el Estado benefactor y la llamada democracia de competencia entre partidos parecían haber logrado la difícil cuadratura del círculo: combinar la economía privada con la participación. Esto no ha sido la consecuencia de un sistema de alianzas interclases o fruto de la cesión de poder al Estado (garante de lo común) por parte de agentes privados. Tal vez fue así en un principio y como consecuencia de las circunstancia económicas irrepetibles. Se trataría más bien de la introducción en la política (lo público) de mecanismo de mercado (lo privado) de tal forma que se ha privatizado la política.

Tal es así que la democracia representativa, siempre controlada a través de los mecanismos partidarios que han taponado la perspectiva de la participación real democrática de las masas, nunca ha planteado graves conflictos que apuntaran a una transformación global del sistema utilizando la vía institucional. En las contadas ocasiones en que esto se ha dado, la mano de hierro oculta en las entretelas del sistema ha aflorado una y otra vez para restablecer el orden, siempre el mismo, -el caso Chileno es paradigmático-.

Tal vez estemos ante un proceso de expropiación que tiene su punto de partida en la propia constitución de los Estados Nacionales, o la historia de los Estados Modernos haya sido la tensión entre dos 
fuerzas contradictorias que Marx definiría como "lucha de clases", y que en otros términos podría expresarse como lo público frenta a lo privado, el interés particular frente al interés colectivo, el Estado frente a la sociedad civil.... Este proceso, entre otros, ha dado lugar a movilizaciones sociales que siguiendo la filosofia de la igualdad y el bien común reclaman cotas de participación democrática como vía para lograr mejoras en sus condiciones de vida. Esto parece ser común a la realidad Europea y la Latinoamericana.

Todo lo anterior permite interpretar los "movimientos sociales" en términos de revindicación de derechos civiles pero matizando que no se trata de una "participación" que se resuelva, tal y como sugieren algunos analistas, con la construcción de canales institucionales que reconduzcan la conflictividad social, por lo menos en el caso de Latinoamérica. Las democracias difícilmente encuentran momento de reposo.

El problema tampoco está en la falta de eficacia del Estado (ese es un término muy relativo que depende del "para qué" de la eficacia). En el caso de los países desarrollados, con el recrudecimiento de la situación económica, la tan nombrada recesión, asistimos a los problemas de variante socialdemócrata. Como el propio Claus Offe afirmara" la perspectiva de una situación de no-crecimiento, destruye la base de relaciones cooperativas entre los actores colectivos, quedan cuestionadas la confianza, el respeto mutuo y la reciprocidad" (op. cit. p. 84).

\section{-La trampa de los conceptos-}

Llegado a este punto cabe preguntarse qué parcela de realidad atrapamos con el concepto de "movimientos sociales".
Los conceptos, en general, no son entes autónomos de la realidad que los da vida, no sólo tienen contenidos específicos sino que deben ser vistos en su historicidad para que realmente puedan sernos de utilidad. No hablamos de un problema de lenguaje. Hablamos de la generalización y universalización teórica y conceptual a la que son tan aficionados los sociólogos y de la "moda" de la comparación que acaba de un plumazo con las especificidades sin encontrar cuales son los niveles válidos de la comparación.

$\mathrm{Si}$ repasamos las teorizaciones sobre movimientos sociales lo primero que llama la atención es el uso indicrimanado del término tanto para hablar de asociaciones formales 0 institucionalizadas $y$ organizaciones estables, como de movimientos espontáneos, sobre todo en el caso de Europa. En Latinoamérica se incluyen movilizaciones obreras, campesinas, etnicas, etc... Hablamos de ecologístas, de pacifistas, de mujeres, de trabajadores, de negros 0 indios... y todos parecen entrar en el mismo saco cuando se habla de movimientos sociales, porque en el fondo la noción clave: "sujeto social" unifica todas estas expresiones alrededor de las ideas de "conciencia y proyecto" (al margen de cuales sean las características de ambos).

Hasta hace poco, la categoría "clase social" había servido más que ninguna otra para responder a determinadas manifestaciones de lo real. Como concepto tiene un largo proceso histórico, desde mediados del siglo XIX, que le va dotando de múltiples contenidos, ya no referidos a la relación del sujeto con los medios de producción sino a los componentes ideológicos. Lo que incorpora el concepto de "movimientos sociales", al margen de los problemas de algunos investigadores para saber de qué sujetos hablan en cada momento, tiene que 
ver con la emergencia de nuevos patrones de acción colectiva. No cesariamente se trata del paso de patrones de legitimación a otros de contestación del orden (a veces es así como característica impuesta por la represión). Más se relaciona con los espacios en los que se sitúa la movilización social y con los ejes alrededor de los cuales se constituye la identidad colectiva. Todo ello no tiene que ver con si son o no los mismos sujetos que en épocas precedentes, eso corresponde a un análisis diferente que converge con el análisis de clase.

La cuestión de los movimientos sociales no puede reducirse a determinadas características estructurales ya sean políticas o económicos pero tampoco puede ser ajena a ellas. Siguen existiendo tendencias detectables en la estructura económica y social que determinan, en parte, las posibles direcciones de la acción social pero que no la explican en su totalidad. Si reducimos las características políticas a los factores económicos perderiamos de vista la gran plasticidad del organismo humano para dar diferentes respuestas frente a un misma situación. Por otro lado los componentes "tiempo" y "espacio" no son ni mucho menos secundarios. Los ritmos por los que los sujetos toman conciencia y pasan a la acción son diferentes a los de los procesos económicos, también difieren en los diferentes espacios culturales.

El tema que aparece en primer plano no es pues la composición, en términos de sujetos, de los "movimientos sociales", sino los procesos de constitución de dichos sujetos, es decir, los mecanismos por los que los individuos se convierten en "sujetos de acción social". Aquí,los problemas de las condiciones de existencia de los individuos (relación respecto de los medios de producción) y el tema de la elaboración subjetiva que hacen de sus condiciones de existencia convergen.
Pongamos un ejemplo, cuando las mujeres se organizan, ya sea en Europa como en Latinoamérica, teniendo como referente explícito su identidad colectiva como mujeres y sus revindicaciones son tan particulares como la supresión de las desigualdades asentadas en las diferencias de género, no podemos usar, o por lo menos no sólo, el concepto de "clase social" para explicar la fuerza motriz que las hace constituirse como sujetos de acción colectiva. Si así lo hiciéramos perderíamos la especificidad del sujeto estudiado. Eso no quiere decir que dichas mujeres no pertenezcan a clases sociales, $\mathrm{e}$ incluso que todas se encuentre en las mismas condiciones objetivas de existencia, o que dichas desigualdades no tengan sus raíces en el patriarcalismo y la dominación masculina unida al capitalismo, ni que debamos definir clase social como un todo compacto al que corresponde una determinada forma de pensar.

La división laboral impuesta por las relaciones capitalistas puede ser fundamental para comprender la explotación que sufren las mujeres, sin embargo, la necesidad percibida por ese conjunto de individuos, lo que les hace constituirse como sujetos de acción colectiva se encuentra en la interpretación subjetiva hecha de esas condiciones objetivas. ¿De qué forma se realiza disha traducción subjetiva, qué o quien interviene en esa elaboración $y$ por qué la identidad colectiva en determinados momentos de la historia se construye en torno a unos valores y no otros? son preguntas que es necesario hacerse para entender las nuevas formas de expresión de los sujetos sociales. Pero sobre todo para no confundir los conceptos con la realidad y para no despreciar instrumentos útiles por nuestra incapacidad para utilizarlos correctamente (aunque mucho nos tememos que se trate más de una opción ideológica). 
A estas alturas, después de la vasta producción intelectual que existe sobre "movimientos sociales", tengo mis dudas acerca de la realidad de la que hablamos, $y$ muchas más sobre el para qué del estudio de este fenómeno, es decir, sobre la finalidad que guía al investigador y que le hace discriminar unos contenidos y no otros.

Nos encontramos, por ejemplo, ante la necesidad de localizar en el espacio y el tiempo el fenómeno encuadrado en el concepto, evitando las generalizaciones metafísicas sin referentes en lo concreto.

Por otro lado necesitamos una sociología comprensiva que sea capaz de determinar el significado y alcance de los cambios que se observan en el comportamiento de los sujetos sociales, capaz de enriquecer los instrumentos conceptuales $y$ no de desecharlos o empobrecerlos. Por eso en nuestro caso concreto hay que dirigir las preguntas al propio objeto de estudio: ¿quién/quiénes son los sujetos de la acción social? y ¿qué es lo que hace que sean sujetos o que no lo sean?. También hemos de bombardear con preguntas al propio investigador ya que de ello depende en parte la elección del marco analítico, y los presupuestos epistemológicos: ¿para qué queremos saber sobre sujetos sociales?.

Es necesario someter a crítica no sólo a las teorías que se utilizan -estén o no explícitassino a los propios conceptos, ya que el concepto empleado y los contenidos apriorísticos incluidos en él nos limitan la capacidad de percibir las variaciones de la realidad, $o$ incluso ver hasta qué punto la realidad que describimos, sobre todo en los análisis comparativos es la misma. A veces no basta con diferenciar los contextos político, económico o cultural porque incluso al interior de un mismo país cuando analizamos un período relativamente largo de tiempo, las variaciones de la realidad que creamos conocer son grandes y ésto implica variaciones de contenido de los conceptos que utilizamos.

No hay "en si" en la historia y, sin embargo, nuestra tendencia es como la de los cuerpos hacia el reposo, una vez que creemos conocer un fenómeno lo disecamos conceptualmente. Es un recurso que utilizamos para poder generalizar. Puede que eso ocurriera con el concepto de clase social y puede que lo mismo esté ocurriendo con el de movimiento social. EI resultado es siempre la esclerotización del conocimiento.

Indudablemente, no nos queda más remedio que servirnos de nuestros pies para andar, y de nuestros conceptos para pensar, pero si no se hace un uso dinámico de los mismos, si no se les obliga a someterse al criterio de la historicidad, puede que estemos creando realidades conceptuales que no van más allá de nuestra propia fantasía. Puede que sea un ejercicio válido pero puede ocurrirnos como al "caballero inexistente" del cuento de Italo Calvino, que llegado el momento de la acción, la realidad le enfrentaba una y otra vez con su in existencia.

Tenemos, por ejemplo, el caso de los que hablan de "los nuevos movimientos sociales" como alternativa a los partidos políticos, como algo autónomo, despolitizado etc., y no se molestan en averiguar que entienden los sujetos por política, que entiende el propio investigador, ni la presencia o no de cuadros de partidos políticos (en general extraparlamentarios) que actúan de dirigentes de dichos movimientos. Luego, hay otros que hacen listados de movimientos sociales identificando el concepto con organizaciones 
formales, inventan entonces sujetos donde no los hay, y el estallido social parece estar a la vuelta de la esquina.

La realidad es un gran generador de preguntas que se emiten desde distintos campos, a veces más importante que elaborar respuestas acabadas, es ser capaz de recoger esas preguntas. Eso puede hacerse abriendo las fronteras de los conceptos, éstos son puntos de partida pero no pueden serlo de llegada.

Cuando trabajamos con estudios realizados por investigadores europeos claramente percibimos que se ha producido una exclusión de los contenidos de clase en el nuevo concepto, cosa que no ocurre en los estudios sobre Latinoamérica. ¿Se trata de dos realidades completamente diferentes? Puede que sí, lo importante de las comparaciones es considerarlas como estrategias útiles a la investigación no como fines en sí mismas.

En otras palabras, hay que "dialectizar" el pensamiento evitando que se estanque y sea un mero reproductor de lugares comunes.

Uno de los supuestos epistemológicos básicos ha de ser el de la realidad no acabada. Si consideramos que el objeto de las ciencias sociales es un conjunto de hechos dados con unas lógicas definitivas y universales, nuestra investigación sobre "sujetos sociales" se dirigirá hacia el descubrimiento de leyes universales que expliquen los hechos en forma de sucesiones causales. La causa del comportamiento de este $u$ otro grupo estudiado estará en función de la correlación de un número concreto de variables predeterminadas. Por el contrario, si pensamos que la realidad no es un todo dado sino un proceso no acabado y en construcción, la causa y el resultado de los fenómenos sociales no se reducirá a la correlación de unas pocas variables sino que será algo más complejo; hablaremos entonces de articulación de procesos. Se abren las puertas hacia el futuro, es decir, hacia lo que pueden llegar a ser o no ser los procesos sociales y a nuestra posibilidad de incidir en ellos.

Estamos hablando de otra forma de razonar, que desde luego no es nueva pero que sí ha sido olvidada unas veces en función de la instrumentalidad técnica, otras para exorcizar a los demonios de la transformación social. Pero que en cualquier caso es un imperativo de la realidad sociohistórica.

Los fenómenos sociales, en cuanto que históricos, pueden tomar distintas direcciones según un abanico de potencialidades que es necesario, esta vez sí, descubrir. En el caso de los "movimientos sociales", lo relevante será descubrir esas potencialidades que más nos interesan para poder incidir en ellas.

El aprendizaje histórico sobre la constitución de sujetos sociales nos obliga a pensar que la realidad está sujeta tanto a la propia acción de los hombres como a los automatismos derivados de estructuras ya cristalizadas. Por tanto, la descripción de esa realidad así como su explicación y la direccionalidad predecible tendrá que ver con nuestra capacidad para captar las estructuras ocultas que marcan sus tendencias, las potencialidades implícitas y la voluntad de los propios sujetos que construyen la realidad. Las consecuencias que extrae el pensamiento social son dos:

a) Apostar por un pensamiento critico y dlaléctico, es decir, no acabado, como la propia realidad; $\mathbf{y}$,

b) La lectura politica de la realidad en función de una opción práctica. 
La relación entre conocimiento y realidad no es univoca como pensara Hegel, sino que está mediada. Por eso descubrir esas mediaciones es tan fundamental como captar la realidad.

Finalmente hay que tener en cuenta que la acción social, entendiendo por tal, la acción de un grupo a favor de la transformación o cambio social, no es lo común sino todo lo contrario, es lo extraordinario, lo que cae fuera de la norma. Como diría Agnes Heller, en las sociedades en que vivimos "la vida cotidiana viene "necesariamente alienada" y el individuo se identifica con los hábitos de su autoconservación que hacen de su vida algo cómodo y falto de conflicto. Así, la toma de conciencia o trascendencia del individuo que le hace en- frentar un proyecto de transformación social (ya sea parcial o global) significa asumir un riesgo. Que haya sujetos que lo asuman, los límites hasta donde pueden llegar y los procesos por los que llegan a elegir esa opción conforman el núcleo problemático de "los movimientos sociales" como objeto de investigación.

Por todo lo anterior, queda claro que la pregunta con la que iniciábamos esta breve reflexión sólo quiere ser una llamada de atención, primero sobre el trabajo intelectual acerca de los movimientos sociales que requiere una reflexión sobre el propio conocimiento, y segundo sobre la tensión necesaria entre lo concreto real que se investiga y la necesidad de teorizar y hacer un uso crítico de las teorías.

\section{RESUMEN}

La autora trata de ubicar la discusión teórica y proponer un nuevo ángulo de lectura de los "movimientos sociales". Piensa que el debate sobre el fenómeno se inscribe en dos coordenadas: La integración social y, la constitución de los sujetos de la acción social. Después de repasar la emergencia de los llamados "movimientos sociales" y rescatar algunos puntos como la cuestión de las identidades y las motivaciones, concluye que el concepto que se utiliza para designar una realidad tan compleja puede llegar a ser una trampa que empobrezca la realidad en vez de dotarla de nuevos contenidos.

\section{ABSTRACT}

The author propose to place the theoretical discussion about social movements. She thinks that problem of social subjet is a matter of: Social integration, and constitution of social subjets. After reviewing the emerging of social movements, she point out the identity and motivations questions. One of the conclusions is that the concept used can be a trap becouse adds new topics but impoverishs the reality at the same time. 